University of Wollongong

Research Online

Faculty of Engineering and Information

Faculty of Engineering and Information

Sciences - Papers: Part A

Sciences

$1-1-2016$

\title{
Nonlocal transistor based on pure crossed Andreev reflection in a EuO- graphene/superconductor hybrid structure
}

Yee Sin Ang

Singapore University of Technology and Design, University of Wollongong, angy@uow.edu.au

Lawrence Ang

Singapore University of Technology and Design

C Zhang

University of Wollongong, czhang@uow.edu.au

Zhongshui Ma

Peking University, Collaborative Innovation Center of Quantum Matter, zma@uow.edu.au

Follow this and additional works at: https://ro.uow.edu.au/eispapers

Part of the Engineering Commons, and the Science and Technology Studies Commons

Research Online is the open access institutional repository for the University of Wollongong. For further information contact the UOW Library: research-pubs@uow.edu.au 


\title{
Nonlocal transistor based on pure crossed Andreev reflection in a EuO-graphene/ superconductor hybrid structure
}

\author{
Abstract \\ We study the interband transport in a superconducting device composed of graphene with EuO-induced \\ exchange interaction. We show that pure crossed Andreev reflection can be generated exclusively without \\ the parasitic local Andreev reflection and elastic cotunnelling over a wide range of bias and Fermi levels in \\ an EuO-graphene/superconductor/EuO-graphene device. The pure nonlocal conductance exhibits rapid \\ on-off switching and oscillatory behavior when the Fermi levels in the normal and the superconducting \\ leads are varied. The oscillation reflects the quasiparticle propagation in the superconducting lead and \\ can be used as a tool to probe the subgap quasiparticle mode in superconducting graphene, which is \\ inaccessible from the current-voltage characteristics. Our results suggest that the device can be used as \\ a highly tunable transistor that operates purely in the nonlocal and spin-polarized transport regime.

\section{Keywords} \\ andreev, reflection, euo, graphene, superconductor, hybrid, structure, nonlocal, pure, transistor, crossed

\section{Disciplines} \\ Engineering | Science and Technology Studies

\section{Publication Details} \\ Ang, Y., Ang, L. K., Zhang, C. \& Ma, Z. (2016). Nonlocal transistor based on pure crossed Andreev reflection \\ in a EuO-graphene/superconductor hybrid structure. Physical Review B: Condensed Matter and Materials \\ Physics, 93 (4), 041422-1-041422-5.
}




\title{
Nonlocal transistor based on pure crossed Andreev reflection in a EuO-graphene/superconductor hybrid structure
}

\author{
Yee Sin Ang, ${ }^{1,2,3}$ L. K. Ang, ${ }^{1,2, *}$ C. Zhang, ${ }^{3, \dagger}$ and Zhongshui $\mathrm{Ma}^{4,5}$ \\ ${ }^{1}$ Engineering Product Development, Singapore University of Technology and Design, Singapore 487372 \\ ${ }^{2}$ SUTD-MIT International Design Center, Singapore University of Technology and Design, Singapore 487372 \\ ${ }^{3}$ School of Physics, University of Wollongong, Wollongong, NSW 2522, Australia \\ ${ }^{4}$ School of Physics, Peking University, Beijing 100871, China \\ ${ }^{5}$ Collaborative Innovation Center of Quantum Matter, Beijing 100871, China
}

(Received 12 November 2015; published 26 January 2016)

\begin{abstract}
We study the interband transport in a superconducting device composed of graphene with EuO-induced exchange interaction. We show that pure crossed Andreev reflection can be generated exclusively without the parasitic local Andreev reflection and elastic cotunnelling over a wide range of bias and Fermi levels in an EuO-graphene/superconductor/EuO-graphene device. The pure nonlocal conductance exhibits rapid on-off switching and oscillatory behavior when the Fermi levels in the normal and the superconducting leads are varied. The oscillation reflects the quasiparticle propagation in the superconducting lead and can be used as a tool to probe the subgap quasiparticle mode in superconducting graphene, which is inaccessible from the current-voltage characteristics. Our results suggest that the device can be used as a highly tunable transistor that operates purely in the nonlocal and spin-polarized transport regime.
\end{abstract}

DOI: 10.1103/PhysRevB.93.041422

Introduction. Andreev reflection (AR) is the excitation of a hole in a normal/superconductor interface when two opposite-spin electrons are coupled into a Cooper pair in the superconductor [1]. In a normal/superconductor/normal $(\mathrm{N} / \mathrm{S} / \mathrm{N})$ three-terminal geometry, two electrons can couple locally in the same normal lead or nonlocally in different normal leads to form a Cooper pair in the superconductor. The local coupling produces the "usual" AR while the nonlocal coupling produces the exotic crossed Andreev reflection (CAR) [2]. The reverse process of CAR in an N/S/N device has been proposed as the basis of a Cooper pair splitter that generates an entangled electron pair in a condensed matter environment [3-7]. High Cooper pair splitting efficiency of $~ 90 \%$ has been experimentally achieved in a carbon-nanotube-based $\mathrm{N} / \mathrm{S} / \mathrm{N}$ device [8]. Moreover, the pairing symmetry of a superconductor can also be probed by a CAR signal [9]. Unfortunately, the generation of CAR-dominated transport is challenging since it is inevitably plagued by electron elastic co-tunnelling (EC) and local AR [10].

Generating pure CAR (pCAR) using energy band topology was first proposed in a graphene bipolar transistor [11]. By precisely tuning the Fermi levels and the bias voltage, EC and local AR excitations are forced to lie exactly on the Dirac points. Due to the vanishing quasiparticle density, EC and AR are completely eliminated. Despite its conceptual simplicity, the experimental realization is difficult since precisely fixed Fermi levels and bias are required. A significant improvement can be achieved by using a gapped energy dispersion [12]. $\mathrm{AR}$ and EC are completely blocked by the whole continuum of the band gap instead of a single Dirac point, thus lifting the constraint on the bias voltage. pCAR mediated by bandgap blocking can occur in semiconductors [12], silicene [13], $\mathrm{MoS}_{2}$ [14], and quantum spin Hall insulators [15], provided

\footnotetext{
*ricky_ang@sutd.edu.sg

†czhang@uow.edu.au
}

that the Fermi levels are placed within one superconducting gap with respect to the conduction and valence band extrema.

The stringent condition of having precisely fixed Fermi levels can be circumvented, for example, by engineering the valley helicity [16] of a zigzag graphene nanoribbon [17] and by shifting the valley-spin splitting [18] in the valence band of $\mathrm{MoS}_{2}$ [19]. In systems with a tunable band gap such as silicene and bilayer graphene [20,21], the existence of a one-dimensional topologically protected edge state [22] provides another opportunity to create widely tunable pCAR. Remarkably, the suppressed intervalley scattering forces a further removal of the normal electron reflection (ER) [23]. Beyond superconductivity, tunable pCAR has been predicted [24] in the topological exciton condensate in threedimensional topological insulators [25]. This offers an exciting alternative condensed matter platform to generate entangled electrons.

Theoretical concept. We propose a different strategy to achieve widely tunable pCAR in this work. We show that the interband transport in a gapped and spin-split energy dispersion can sustain pCAR over a wide range of bias and Fermi levels. The concept is illustrated in Fig. 1(a). Consider the case where the Fermi level of the incident side, $E_{F, 1}$, is placed between the two conduction spin-subband edges and that of the transmitted side, $E_{F, 2}$, is placed between the two valence spin-subband edges. For an incident electron residing in the lower conduction spin subband, no oppositespin electrons are available below the Fermi level for local AR. In the transmitted region, spin conservation forbids the electron from tunneling into the opposite-spin valence subband. As a result, the only permissible processes are ER and the much sought-after pCAR. The conditions of having precisely tuned bias and Fermi levels are both relaxed. To demonstrate this, we consider a europium oxide-graphene (EuO-G) ferromagnetic hybrid structure [26] [Fig. 1(b)]. First-principles calculations predicted that $\mathrm{EuO}$ strongly spin polarizes the $\pi$ orbitals of graphene $[27,28]$ and induces a large exchange splitting. 
(a)

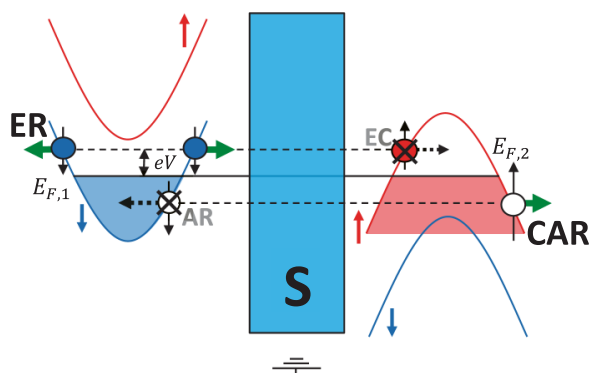

(b)

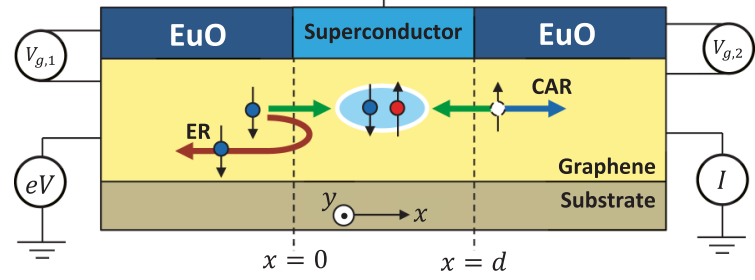

FIG. 1. (a) Mechanism of pCAR in a gapped and spin-split dispersion. (b) Schematic of the EuO-G/superconductor/EuO-G device. The incident energy is related to the bias by $E=e V$.

A sizable spin-dependent band gap, which crucially blocks the local AR and EC excitations, is present. We found that the nonlocal conductance in EuO-G/S/EuO-G exhibits fast on-off switching via normal leads gating. Furthermore, the nonlocal conductance exhibits an oscillatory behavior with the superconductor gate that directly reflects the subgap superconducting Dirac quasiparticle propagation. We found that a minimal subthreshold swing of $15.1 \mathrm{mV}$ and a large onoff ratio of $10^{5}$ can be achieved. This opens up the possibility of a high efficiency graphene-based nonlocal transistor in which all local and nonentangled processes are suppressed.

Model. In $\mathrm{EuO} / \mathrm{G}$, the $K$ and $K^{\prime}$ Dirac cones are mapped onto the $\Gamma$ point due to the Brillouin zone folding $[27,28]$. The low energy effective Hamiltonian can be written as [29] $\mathcal{H}_{\boldsymbol{k}, \sigma}=\sigma h \mathcal{I}+\Delta_{\sigma} \tau_{z}+\hbar v_{\sigma} \boldsymbol{k} \cdot \boldsymbol{\tau}$, where $\sigma= \pm 1$ for spin-up and spin-down electrons, $h$ is the proximity-induced exchange interaction, $\boldsymbol{k}=\left(k_{x}, k_{y}\right)$ is the electron wave vector, $\boldsymbol{\tau}=$ $\left(\tau_{x}, \tau_{y}, \tau_{z}\right)$ are the Pauli matrices, and $v_{\sigma}$ and $\Delta_{\sigma}$ are the spin-dependent Fermi velocity and band gap, respectively. $\mathcal{I}$ is a $2 \times 2$ identity matrix. The eigenenergy is $\varepsilon_{\sigma \eta}(\boldsymbol{k})=$ $\eta \sqrt{\Delta_{\sigma}^{2}+\hbar v_{\sigma}^{2} k^{2}}+\sigma h$ where $k=|\boldsymbol{k}|$ and $\eta= \pm 1$ denotes conduction and valence bands. The normalized eigenstate is $\xi_{\sigma \eta}(\boldsymbol{k})=\left[\left(\varepsilon_{\sigma \eta}(\boldsymbol{k})-\Delta_{\sigma}\right) / 2 \varepsilon_{\sigma \eta}(\boldsymbol{k})\right]^{1 / 2}\left[\frac{\hbar v_{\sigma} k_{x}-i k_{y}}{\varepsilon_{\sigma \eta}(\boldsymbol{k})-\Delta_{\sigma}}, 1\right]^{T}$, where $T$ stands for transpose. First-principles calculation [27] gives $v_{\sigma}=(1.4825-0.1455 \sigma) \times v_{F}, h=31 \mathrm{meV}$, and $\Delta_{\sigma}=$ $(58+9 \sigma) \mathrm{meV}$. The Bogoliubov-de Gennes equation [30] is given as

$$
\left(\begin{array}{cc}
\mathcal{H}_{k, \sigma}(x)-E_{F} \mathcal{I} & \Delta_{s c}(x) \mathcal{I} \\
\Delta_{s c}^{*}(x) \mathcal{I} & -\left(\mathcal{H}_{k, \bar{\sigma}}(x)-E_{F} \mathcal{I}\right)
\end{array}\right)\left(\begin{array}{c}
u_{\sigma} \\
v_{\bar{\sigma}}
\end{array}\right)=E_{\sigma}(\mathbf{k})\left(\begin{array}{c}
u_{\sigma} \\
v_{\bar{\sigma}}
\end{array}\right)
$$

where $\bar{\sigma}=-\sigma, h(x)=h$ for $0>x>d$, and the superconducting gap is $\Delta_{s c}(x)=\Delta_{s c}$ for $0<x<d$. We take the phase in $\Delta_{s c}$ as zero. For $\Delta_{s c}(x)=0$, Eq. (1) can be decoupled into a spin- $\sigma$ electron part and a spin- $\bar{\sigma}$ hole part. As $k_{y}$ is a good quantum number, we write $k_{ \pm}=-i \partial / \partial x \pm i k_{y}$ and solve Eq. (1) for the quasiparticle eigenstates and the
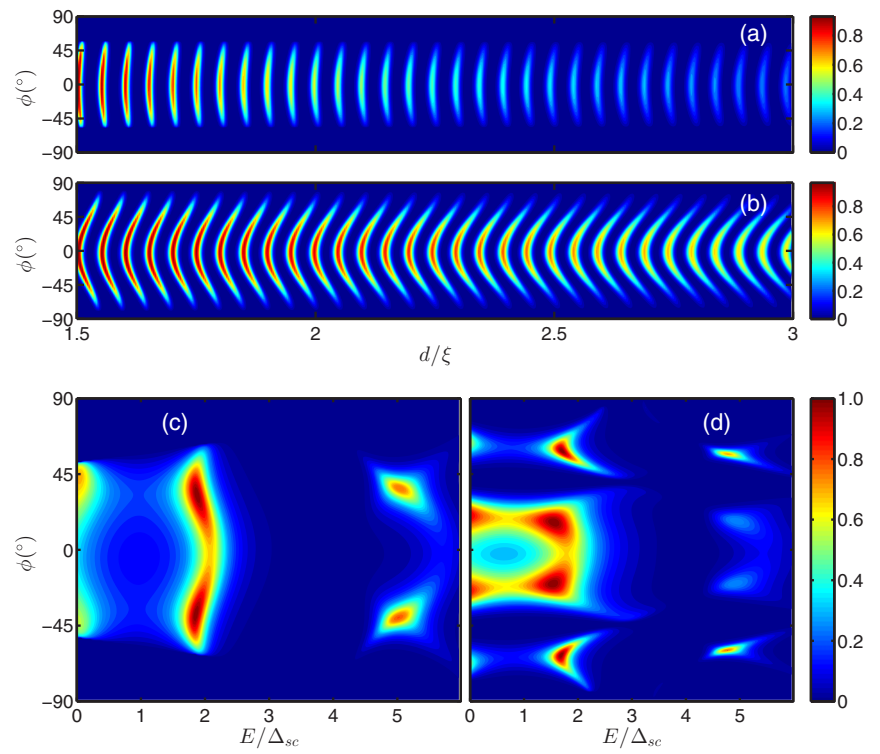

FIG. 2. $T_{C A R}$ as a function of the superconductor width, $d$, for (a) $\left(E_{F, 1}, E_{F, 2}\right)=(30,-40) \mathrm{meV}$; and (b) $\left(E_{F, 1}, E_{F, 2}\right)=(60,-60) \mathrm{meV}$ with $E / \Delta_{s c}=0.9\left(\xi=\hbar v_{F} / \pi \Delta_{s c}\right)$. (c) and (d) shows the $T_{C A R}$ as a function of incident energy $E / \Delta_{s c}$ at the same Fermi levels as (a) and (b), respectively, with $d=2.5 \xi\left(\Delta_{s c}=1 \mathrm{meV}\right.$ and $\left.\mu_{S}=200 \mathrm{meV}\right)$.

excitation energies. The transport coefficients can then be straightforwardly obtained by matching the wave functions at $x=0$ and at $x=d$ [31].

Results and discussions. In the numerical calculation, we choose $\Delta_{s c}=1 \mathrm{meV}$, which agrees with a recent experimental value [32]. For conciseness, we focus on the pCAR transport phenomenon originating from an incident electron residing in the $\sigma=-1$ conduction subband and transmitted as a purely $\sigma=+1$ polarized valence hole. Since there is a large common gap of $\left(\Delta_{+}+\Delta_{-}-2 h\right)=54 \mathrm{meV}$, only a conduction hole is involved in the quasiparticle transport [33]. According to first-principles results [27,29], the Fermi levels lie in the ranges of $18 \mathrm{meV}<E_{F, 1}<98 \mathrm{meV}$ and $-80 \mathrm{meV}$ $<E_{F, 2}<-36 \mathrm{meV}$. This corresponds to wide windows of $\Delta E_{F}^{(c)}=80 \mathrm{meV}$ and $\Delta E_{F}^{(v)}=44 \mathrm{meV}$ for conduction and valence bands, respectively. We first study the pCAR transmission probabilities, $T_{C A R}$, in Fig. 2. The angle of incidence of the electron is denoted by $\phi . T_{C A R}$ oscillates rapidly with $d$ because of the quasiparticle interference in the superconducting gap [Fig. 2(a)]; transmission peaks occur whenever the subgap superconducting quasiparticle wave vector matches the resonance wave vector $k_{0}=n \pi / d$. A significant difference between Figs. 2(a) and 2(b) is that the resonance "stripes" are almost vertical and well separated in Fig. 2(a) (Fermi levels lie closer to the band edges), while in Fig. 2(b) the resonance patterns are curved and are no longer well separated (Fermi levels lie farther away from the band edges). This contrasting behavior can be seen in the $\mathrm{eV}$ dependence of $T_{C A R}$. Along a vertical cut at $E / \Delta_{s c}=2$ and $E / \Delta_{s c}=5$, four $T_{C A R}$ hot spots are clearly present in Fig. 2(d) instead of only two in Fig. 2(c). The four hotspot is caused by the curved resonance pattern in Fig. 2(b) as it is composed of two pairs of transmission resonance at constant $d / \xi$ : one from 

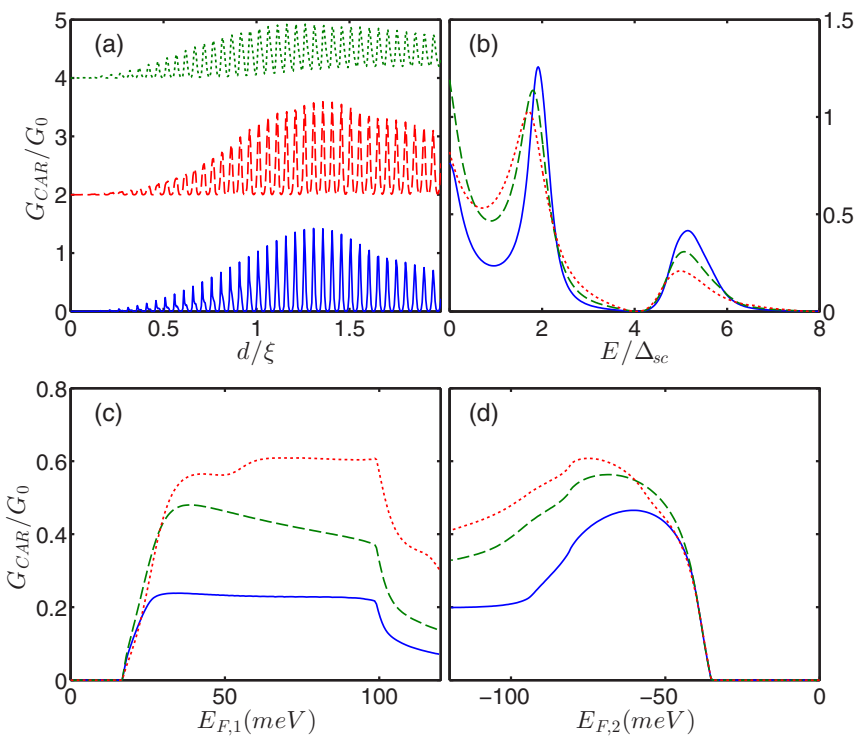

FIG. 3. Nonlocal conductance $G_{C A R}$. (a) $d$ dependence for (solid) $\left(E_{F, 1}, E_{F, 2}\right)=(30,-40) \mathrm{meV}, \quad$ (dashed) $\quad\left(E_{F, 1}, E_{F, 2}\right)=(30,-$ $60) \mathrm{meV}$, and (dotted) $\left(E_{F, 1}, E_{F, 2}\right)=(60,-60) \mathrm{meV}$ at $E / \Delta_{s c}=0.9$ (data are offset vertically by $2 G_{0}$ for clarity and $E=0.9 \Delta_{s c}$.); (b) $E / \Delta_{s c}$ dependence with the same Fermi levels as (a) and $d=$ $2.5 \xi$; (c) $E_{F, 1}$ dependence with $E_{F, 2}=-40,-50,-70 \mathrm{meV}$ (solid, dashed, and dotted line, respectively); and (d) $E_{F, 2}$ dependence with $E_{F, 1}=30,40,60 \mathrm{meV}$ (solid, broken, and dotted line, respectively). $d=2.5 \xi$ and $E / \Delta_{s c}=0.9$ for (c) and (d). When $E_{F, 1}>98 \mathrm{meV}$ and $E_{F, 2}<-80 \mathrm{meV}, G_{C A R}$ decreases significantly due to the onset of the competing local AR and EC processes.

the central region of a resonance stripe and one from the tail region of the preceding curved resonance stripe.

The zero-temperature nonlocal conductance generated by pCAR is given as [34] $G_{C A R} / G_{0}=\int T_{C A R} \cos \phi d \phi . G_{0}$ is the ballistic normal conductance in the $\sigma=-1$ channel. In Fig. 3(a), $G_{C A R}$ as a function of the superconductor width, $d$, is plotted. $G_{C A R}$ oscillates rapidly with $d$ due to the fast oscillation of $T_{C A R}$. Interestingly, $G_{C A R}$ minima are near zero only when the $E_{F}$ 's are close to the band edge. This is a direct consequence of the well-separated resonance stripes as discussed in Fig. 2(a). In Fig. 3(b), the $G_{C A R}$ resonances occur at $E / \Delta_{s c} \approx 2$ and $\approx 5$. This is consistent with the $T_{C A R}$ hot spots observed in Figs. 2(c) and 2(d). Note that although the $T_{C A R}$ peaks originates from the Fabry-Perot interference (FP) inside $\Delta_{s c}$, the tunneling current of solely pCAR is unachievable via FP alone since $G_{C A R}$ is an angular-averaged quantity. The selective enhancement of one transport process and the simultaneous suppression of the rest is only achievable at certain incident angles for a given energy. Without filtering out the local AR and EC processes via the band topology, the tunneling conductance is inevitably mixed with local and nonentangled components. In the proposed device, $\left(E_{F, 1}, E_{F, 2}\right)$ can be tuned without destroying the pCAR. $G_{C A R}$ as a function of $\left(E_{F, 1}, E_{F, 2}\right)$ is calculated in Figs. 3(c) and 3(d). The onset of $G_{C A R}$ for incident energy $E$ is $(18-E) \mathrm{meV}$ and $(-36+E) \mathrm{meV}$ for $E_{F, 1}$ and $E_{F, 2}$, respectively. Before these onsets, $G_{C A R}$ is completely switched off due to the depletion of the charge

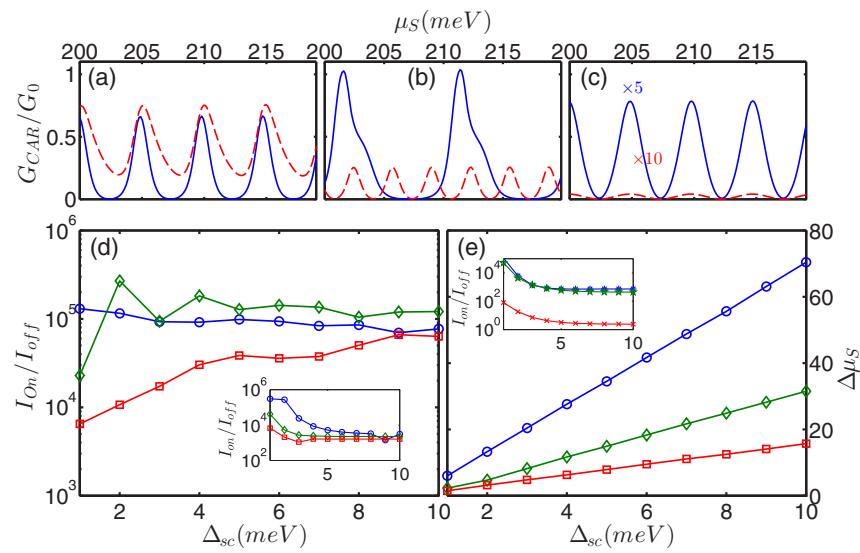

FIG. 4. $\mu_{S}$ dependence of $G_{C A R}$ with $E / \Delta_{s c}=0.9$. (a) Fermi levels $\left(E_{F, 1}, E_{F, 2}\right)$ are $(30,-40) \mathrm{meV}$ (solid) and $(60,-60) \mathrm{meV}$ at $d=2 \xi$ (dashed); (b) $d=\xi$ (solid) and $d=3 \xi$ (dashed); (c) $\Delta_{s c}=$ $2 \mathrm{meV}$ (solid) and $\Delta_{s c}=5 \mathrm{meV}$ (dashed) with $d_{0}$ fixed at $\hbar v_{F} / \tilde{\Delta}_{s c}$ where $\tilde{\Delta}_{s c}=1 \mathrm{meV}$. In (b) and (c), $\left(E_{F, 1}, E_{F, 2}\right)=(30,-40) \mathrm{meV}$ are used. The $\Delta_{s c}$ dependence of (d) $I_{\text {on }} / I_{\text {off }}$ and (e) $\Delta \mu_{s}$ with $E / \Delta_{s c}=$ 0.9 and $\left(E_{F, 1}, E_{F, 2}\right)=(18,-36) \mathrm{meV}$. The widths are $d=\xi(\circ)$, $d=2 \xi(\diamond)$, and $d=3 \xi(\square)$. The inset in (d) is the same as the main plot except that $d=d_{0}(\circ), d=2 d_{0}(\diamond)$, and $d=3 d_{0}(\square)$. The inset in (e) shows the $I_{\mathrm{on}} / I_{\mathrm{off}}$ at several sets of $\left(\Delta_{+}, \Delta_{-}, h\right)$, i.e., $(170,150,90) \mathrm{meV}(*),(210,190,10) \mathrm{meV}(\star)$, and $(30,10,2) \mathrm{meV}$ $(\times) . E / \Delta_{s c}=0.9, d=d_{0}$, and the Fermi levels are fixed at the band edges.

carriers. Remarkably, $G_{C A R}$ rises very sharply post onset, suggesting a potential in fast on-off switching application. To estimate the switching characteristic, we define the Fermi level subthreshold swing as $S S\left(E_{F, i}\right)=\left(d \log _{10} I_{C A R} / d E_{F, i}\right)^{-1} \approx$ $\left(\Delta \log _{10} G_{C A R} / \Delta E_{F, i}\right)^{-1}$ where $i=1,2$ denotes the two normal leads. We found that in the linear-growth regime immediately after the onset, $S S\left(E_{F, 1}\right)$ is about $7.1 \mathrm{meV} / \mathrm{dec}$ (meV per decade). For $E_{F, 2}$, the onset of $G_{C A R}$ is even sharper, yielding $S S\left(E_{F, 2}\right) \approx 3.3 \mathrm{meV} / \mathrm{dec}$. The gate-voltage subthreshold swing, $S S\left(V_{g, i}\right)=\left(d \log _{10} I_{C A R} / d V_{g, i}\right)^{-1}$, can be estimated from experimental data [35,36]. We found that [37] $S S\left(V_{g, 1}\right) \approx 60.5 \mathrm{mV} / \mathrm{dec}$ and $S S\left(V_{g, 2}\right) \approx 15.1 \mathrm{mV} / \mathrm{dec}$. The remarkably small $S S\left(V_{g, 2}\right)$ shows an even steeper on-off switching characteristic in comparison with state-of-the-art $\mathrm{MoS}_{2}$-based transistor recently reported in [38]. This reveals the potential of the proposed device as a fast switching transistor that operates uniquely in the nonlocal and $100 \%$ spin-polarized transport regime.

We calculated $G_{C A R}$ as a function of the superconducting graphene Fermi level, $\mu_{S}$, in Figs. 4(a)-4(c). In general, $G_{C A R}$ exhibits oscillatory behavior with $\mu_{S}$. The following are observed: (i) the oscillation frequency is unaffected by $\left(E_{F, 1}, E_{F, 2}\right)$ [Fig. 4(a)]; (ii) the frequency of $G_{C A R}$ oscillation is reduced by a smaller $d$ [Fig. 4(b)]; and (iii) the oscillation frequency is unaffected by $\Delta_{s c}$ but the amplitude is severely damped at larger $\Delta_{s c}$ [Fig. 4(c)]. These oscillatory behaviors reflect the subgap $\left(E<\Delta_{s c}\right)$ quasiparticle dynamics residing in the superconducting graphene. For $E<\Delta_{s c}$, the superconducting Dirac quasiparticle wave vector is composed of a propagating (real) term, $k_{S}=\sqrt{\left(\hbar v_{F}\right)^{-2} \mu_{S}^{2}+q^{2}}$, and an imaginary (damping) term, $\kappa=\left(\Delta_{s c} / \hbar v_{F} k_{S}\right) \sin \left[\cos ^{-1}\left(E / \Delta_{s c}\right)\right]$ [33]. 
$\mu_{S}$ tuning directly modifies $k_{S}$. When $k_{S}$ is tuned across two successive subgap standing-wave modes, a peak-valley-peak $G_{C A R}$ oscillation is produced. $\left(E_{F, 1}, E_{F, 2}\right)$ do not play a direct role in $k_{S}$. Hence, they do not alter the oscillation frequency [Fig. 4(a)]. When $d$ is decreased, the difference between two successive standing-wave vectors becomes larger as the quantized standing-wave vector $k_{0} \propto 1 / d$. The peak-to-peak transition thus requires a larger range of $\mu_{S}$ to be scanned across. This results in a reduced oscillation frequency as seen in Fig. 4(b). $\Delta_{s c}$ affects only the damping term as $\kappa \propto \Delta_{s c}$ when $E / \Delta_{s c} \rightarrow 1$. Increasing $\Delta_{s c}$ thus leads to a stronger damping which reduces the amplitude without changing its oscillation frequency. Physically, one can interpret the $\Delta_{s c}$ dependence as follows: a larger $\Delta_{s c}$ leads to a shorter coherence length $\xi \propto 1 / \Delta_{s c}$. At a fixed $d_{0}$, the "effective" barrier width becomes larger in the relative sense of $d_{0} / \xi$. Therefore, the CAR tunneling current is heavily damped as the nonlocal Cooper pairing of electrons has to overcome too many coherence lengths.

The $G_{C A R}$ oscillation offers an additional tunable parameter to control the transport by gating the superconducting graphene. As $\mu_{S}$ tuning cannot completely switch the $G_{C A R}$ off and $G_{C A R}$ oscillates periodically, we define two quantities to characterize the $\mu_{S}$-switching effect: (i) the nonlocal current on-off ratio, $I_{\mathrm{on}} / I_{\mathrm{off}} \approx G_{C A R}^{(\max )} / G_{C A R}^{(\mathrm{min})}$ for small bias where $G_{C A R}^{(\max )}$ and $G_{C A R}^{(\min )}$ are the maximum and minimum conductance determined at the vicinity of $\mu_{S} \approx 200 \mathrm{meV}$, respectively; and (ii) the range of $\mu_{S}$ required for peak-to-valley switching, $\Delta \mu_{S}$. For small $d, I_{\mathrm{on}} / I_{\mathrm{off}}$ can be as high as $10^{5}$ over a wide range of $\Delta_{s c}$ [Fig. 4(d)]. Large $\Delta \mu_{S}$ is desirable for efficient $\mu_{S}$ switching so that the valley-to-peak transition is robust against the Fermi level fluctuation induced by charge inhomogeneity and substrate [39]. The $\Delta \mu_{S}$ is in the undesirably small values of few meV at small $\Delta_{s c}$ due to the rapid $G_{C A R}$ oscillation. Interestingly, $\Delta \mu_{S}$ increases linearly with $\Delta_{s c}[$ Fig. 4(e)] and can be improved to $70 \mathrm{meV}$ at $\Delta_{s c}=10 \mathrm{meV}$. The linear relation between $\Delta \mu_{S}$ and $\Delta_{s c}$ can be explained by noting that $d$ is in the unit of $\xi \propto 1 / \Delta_{s c}$ and hence is not fixed in Figs. 4(d) and 4(e). Since the oscillation frequency is determined by the quantized standing-wave vector which is $k_{0} \propto d$ and $d \propto 1 / \Delta_{s c}$, we have $k_{0} \propto \Delta_{s c}$, which leads to the linear dependence. In the insets, we calculated $I_{\text {on }} / I_{\text {off }}$ as a function of $\Delta_{s c}$ with $d$ in the unit of fixed unit $d_{0}=\hbar v_{F} / \pi \Delta_{0}$ where $\Delta_{0}=1 \mathrm{meV}$ [Fig. 4(d)] and for various $\Delta_{\sigma}$ and $h$ at $d=d_{0}$ [Fig. 4(e)]. We observe that $I_{\text {on }} / I_{\text {off }}$ is significantly reduced at small $\Delta_{\sigma}$ and at large $\Delta_{s c}$. This confirms the importance of a large spin-dependent band gap and finite $h$ in achieving efficient nonlocal current gating. Furthermore, strong Cooper pairing does not lead to enhanced nonlocal transport.

Conclusion. In conclusion, we proposed widely tunable pCAR in the interband transport of spin-split and gapped dispersion in EuO-G/S/EuO-G. The proposed device exhibits rapid on-off switching which can potentially be used as a building block in CAR-based quantum computing and spintronics. We emphasize that the pCAR mechanism proposed here is fundamentally different from the case of $\mathrm{MoS}_{2}$ with exchange interaction [19]. In our scheme, pCAR is based on the interband quasiparticle transport between the conduction and the valence spin-split subbands via Fermi levels tuning. Due to the inverted band topology between conduction and valence spin-split subbands, the elimination of the local AR and EC branches can be straightforwardly achieved without the need to shift the relative separation between the subbands via exchange interaction [19]. For EuO-G/S single interface, local AR is completely suppressed in the regime studied here. As it is well known that local AR generates Joule heating that undesirably lowers the cooling power of a normal/insulator/superconductor-based electronic refrigerator [40-42], we expect EuO-G/S to exhibit enhanced sub-Kelvin cooling performance. One major challenge to observe the rapid $G_{C A R}$ oscillation is the fabrication of a high-quality sample as the interface roughness and Fermi level fluctuations can wash out the oscillation. Finally, we point out that the pCAR mechanism proposed here is universally applicable to systems with similar band topology such as YiG-graphene [43] and monolayer transition-metal dichalcogenides with magnetic doping [44] or with proximity to $\mathrm{EuO}$ [45]. These structures offer alternative platforms to test the validity of our prediction.

Acknowledgments. We thank Shi-Jun Liang and Kelvin J. A. Ooi for their fruitful discussions. Z.S.M. is thankful for the support of NSFC (Grant No. 11274013) and NBRP of China (Grant No. 2012CB921300). This work was funded by the Singapore Ministry of Education (MOE) T2 grant ( Grant No. T2MOE1401), and the Australian Research Council Discovery Grant (Grant No. DP140101501).
[1] A. F. Andreev, J. Expt. Theor. Phys. 19, 1228 (1964).

[2] J. M. Byers and M. E. Flatte, Phys. Rev. Lett. 74, 306 (1995).

[3] P. Samuelsson, E. V. Sukhorukov, and M. Buttiker, Phys. Rev. Lett. 91, 157002 (2003).

[4] P. Samuelsson, E. V. Sukhorukov, and M. Buttiker, New J. Phys. 7, 176 (2005).

[5] E. Prada and F. Sols, Eur. Phys. J. B 40, 379 (2004).

[6] G. B. Lesovik, T. Martin, and G. Blatter, Eur. Phys. J. B 24, 287 (2001).

[7] A. Schroer, P. G. Silvestrov, and P. Recher, Phys. Rev. B 92, 241404 (2015).

[8] J. Schindele, A. Baumgartner, and C. Schönenberger, Phys. Rev. Lett. 109, 157002 (2012)

[9] C. Benjamin, Phys. Rev. B 74, 180503(R) (2006).
[10] G. Falci, D. Feinberg, and F. W. J. Hekking, Europhys. Lett. 54, 255 (2001).

[11] J. Cayssol, Phys. Rev. Lett. 100, 147001 (2008).

[12] M. Veldhorst and A. Brinkman, Phys. Rev. Lett. 105, 107002 (2010).

[13] J. Linder and T. Yokoyama, Phys. Rev. B 89, 020504(R) (2014).

[14] C. Bai, Y. Zou, W.-K. Lou, and K. Chang, Phys. Rev. B 90, 195445 (2014).

[15] W. Chen, R. Shen, L. Sheng, B. G. Wang, and D. Y. Xing, Phys. Rev. B 84, 115420 (2011).

[16] A. Cresti, G. Grosso, and G. P. Parravicini, Phys. Rev. B 77, 233402 (2008); A. R. Akhmerov, J. H. Bardarson, A. Rycerz, and C. W. J. Beenakker, ibid. 77, 205416 (2008); A. Rycerz, J. Tworzydlo, and C. W. J. Beenakker, Nat. Phys. 3, 172 (2007). 
[17] J. Wang and S. Liu, Phys. Rev. B 85, 035402 (2012).

[18] D. Xiao, G.-B. Liu, W. Feng, X. Xu, and W. Yao, Phys. Rev. Lett. 108, 196802 (2012); N. Alidoust et al., Nat. Commun. 5, 4673 (2014).

[19] L. Majidi and R. Asgari, Phys. Rev. B 90, 165440 (2014).

[20] N. D. Drummond, V. Zoólyomi, and V. I. Fal'ko, Phys. Rev. B 85, 075423 (2012); Z. Ni, Q. Liu, K. Tang, J. Zheng, J. Zhou, R. Qin, Z. Gao, D. Yu, and J. Lu, Nano Lett. 12, 113 (2012).

[21] Y. Zhang, T.-T. Tang, C. Girit, Z. Hao, M. C. Martin, A. Zettl, M. F. Crommie, Y. R. Shen, and F. Wang, Nature (London) 459, 820 (2009); J. B. Oostinga, H. B. Heersche, X. Liu, A. F. Morpurgo, and L. M. K. Vandersypen, Nat. Mater. 7, 151 (2007); E. V. Castro, K. S. Novoselov, S. V. Morozov, N. M. R. Peres, J. M. B. dos Santos, J. Nilsson, F. Guinea, A. K. Geim, and A. H. Castro Neto, Phys. Rev. Lett. 99, 216802 (2007).

[22] Ivar Martin, Ya. M. Blanter, and A. F. Morpurgo, Phys. Rev. Lett. 100, 036804 (2008); S. K. Wang, J. Wang, and K. S. Chan, New J. Phys. 16, 045015 (2014).

[23] J. Wang, L. Hao, and K. S. Chan, Phys. Rev. B 91, 085415 (2015).

[24] M. Veldhorst, M. Hoek, M. Snelder, H. Hilgenkamp, A. A. Golubov, and A. Brinkman, Phys. Rev. B 90, 035428 (2014).

[25] B. Seradjeh, J. E. Moore, and M. Franz, Phys. Rev. Lett. 103, 066402 (2009).

[26] D. F. Förster, T. O. Wehling, S. Schumacher, A. Rosch, and T. Michely, New J. Phys. 14, 023022 (2012); A. G. Swartz, P. M. Odenthal, Y. Hao, R. S. Ruoff, and R. K. Kawakami, ACS Nano 6, 10063 (2012).

[27] H. X. Yang, A. Hallal, D. Terrade, X. Waintal, S. Roche, and M. Chshiev, Phys. Rev. Lett. 110, 046603 (2013).

[28] S. Su, Y. Barlas, and R. K. Lake, arXiv:1509.06427.

[29] Y. Song and G. Dai, Appl. Phys. Lett. 106, 223104 (2015).

[30] P. G. de Gennes, Superconductivity in Metals and Alloys (Benjamin, New York, 1966); N. N. Bogoliubov, J. Expt. Theor.
Phys. 34, 58 (1958); J. G. Valatine, Nuovo Cimento 7, 843 (1958).

[31] See Supplemental Material at http://link.aps.org/supplemental/ 10.1103/PhysRevB.93.041422 for the derivation of the transport coefficients.

[32] D. K. Efetov et al., Nat. Phys., doi:10.1038/nphys3583 (2015).

[33] C. W. J. Beenakker, Phys. Rev. Lett. 97, 067007 (2006); Rev. Mod. Phys. 80, 1337 (2008); M. Titov and C. W. J. Beenakker, Phys. Rev. B 74, 041401(R) (2006).

[34] G. E. Blonder, M. Tinkham, and T. M. Klapwijk, Phys. Rev. B 25, 4515 (1982).

[35] C.-F. Chen et al., Nature (London) 471, 617 (2011).

[36] S.-F. Shi, T.-T. Tang, B. Zeng, L. Ju, Q. Zhou, A. Zettl, and F. Wang, Nano Lett. 14, 1578 (2014).

[37] Assuming that graphene is undoped, $V_{g}$ follows the relation $E_{F}=346 \sqrt{V_{g}}(\mathrm{meV})$ (See Supplemental Material of [36]). The conversion of $S S\left(E_{F}\right) \rightarrow S S\left(V_{g}\right)$ is performed via the transformation $S S^{-1}\left(V_{g}\right)=d \log _{10} I_{0} / d V_{g}=d \log _{10} I_{0} / d E_{F} \times$ $d E_{F} / d V_{g} \approx S S^{-1}\left(E_{F}\right) \times\left(346 / 2 \bar{E}_{F}\right)$ where $\bar{E}_{F}$ is the average Fermi level.

[38] D. Sarkar, X. Xie, W. Liu, W. Cao, J. Kang, Y. Gong, S. Kraemer, P. M. Ajayan, and K. Banerjee, Nature (London) 526, 91 (2015).

[39] J. Xue, J. Sanchez-Yamagishi, D. Bulmash, P. Jacquod, A. Deshpande, K. Watanabe, T. Taniguchi, P. Jarillo-Herrero, and B. J. LeRoy, Nat. Mater. 10, 282 (2011).

[40] M. Nahum, T. M. Eiles, and J. M. Martinis, Appl. Phys. Lett. 65, 3123 (1994).

[41] A. Bardas and D. Averin, Phys. Rev. B 52, 12873 (1995).

[42] M. M. Leivo, J. P. Pekola, and D. V. Averin, Appl. Phys. Lett. 68, 1996 (1996).

[43] Z. Wang, C. Tang, R. Sachs, Y. Barlas, and Jing Shi, Phys. Rev. Lett. 114, 016603 (2015)

[44] Y. C. Cheng, Q. Y. Zhang, and U. Schwingenschlögl, Phys. Rev. B 89, 155429 (2014).

[45] J. Qi, X. Li, Q. Niu, and J. Feng, Phys. Rev. B 92, 121403(R) (2015). 